\title{
Accuracy versus Performance in Lattice Boltzmann BGK Simulations of Systolic Flows
}

\author{
Abdel Monim Artoli, Lilit Abrahamyan^, and Alfons G. Hoekstra \\ Section Computational Science, Laboratory for Computing, System Architecture and \\ Programming,Faculty of Science, \\ University of Amsterdam \\ Kruislaan 403, 1098 SJ Amsterdam, The Netherlands \\ \{artoli, labraham, alfons\} @science.uva.nl \\ http://www.science.uva.nl/research/scs/
}

\begin{abstract}
The aim of this work is to tune the lattice Boltzmann BGK (LBGK) simulation parameters in order to achieve optimum accuracy and performance for time dependent flows. We present detailed analysis of the accuracy and performance of LBGK in simulating pulsatile Newtonian flow in a straight rigid 3D tube. We compare the obtained velocity profiles and shear stress to the analytic Womersley solutions. A curved boundary condition is used for the walls and the accuracy and performance are compared to that obtained by using the bounce-back on the links. A technique to reduce compressibility errors during simulations based on reducing the Mach number is presented.
\end{abstract}

Keywords: Lattice Boltzmann Method, Systolic Cycle, Abdominal Aorta.

\section{Introduction}

Suitability and accuracy of the newly established lattice Boltzmann method in simulating time dependent fluid flows is demonstrated in the literature 123. It is shown that use of curved boundary conditions noticeably enhances the accuracy as compared to using the simple bounce-back on the links [4.5].

The aim of this study is to end up with optimal simulation parameters for a desired accuracy with minimum simulation time. Simulation parameters for fixed Reynolds and Womersley parameters are studied. The paper is organized as follows: First, we briefly review the LBGK model we are using and then, we discuss the convergence behavior under different simulation choices and set up the optimal conditions for best performance.

\section{The Lattice Boltzmann BGK Method}

The method is based on a discretized Boltzmann equation with simplified collision operator via the single particle relaxation time approximation proposed by

\footnotetext{
* Corresponding author.
} 
Bhatnagar, Gross and Krook in 1954. 6] The LBGK scheme is based on two steps; streaming to the neighboring nodes and colliding with local node populations which are represented by the probability $f_{i}$ of a particle moving with a velocity $\mathbf{e}_{i}$ per unit time step $\delta t$. Populations, which are assumed Maxwellians, relax towards equilibrium during a collision process. The equilibrium distribution function

$$
f_{i}=w_{i} \rho\left(1+\frac{3}{v^{2}} \mathbf{e}_{i} \cdot \mathbf{u}+\frac{9}{2 v^{4}}\left(\mathbf{e}_{i} \cdot \mathbf{u}\right)^{2}-\frac{3}{2 v^{2}} \mathbf{u} \cdot \mathbf{u}\right),
$$

is a low Mach number approximation to the expansion of the Maxwellian distribution. Here, $w_{i}$ is a weighting factor, $v=\delta x / \delta t$ is the lattice speed, and $\delta x$ and $\delta t$ are the lattice spacing and the time step, respectively. [7] The well-known lattice BGK equation

$$
f_{i}\left(\mathbf{x}+\mathbf{e}_{i} \delta_{t}, \mathbf{e}_{i}, t+\delta_{t}\right)-f_{i}\left(\mathbf{x}, \mathbf{e}_{i}, t\right)=-\frac{1}{\tau}\left[f_{i}\left(\mathbf{x}, \mathbf{e}_{i}, t\right)-f_{i}^{(0)}\left(\mathbf{x}, \mathbf{e}_{i}, t\right)\right]
$$

can be obtained by discretizing the evolution equation of the distribution functions in the velocity space using a finite set of velocities $\mathbf{e}_{i}$. In this equation, $\tau$ is the dimensionless relaxation time.In this article, we used the standard quasi incompressible D3Q19 model which has three types of particles on each node; a rest particle, six particles moving along $x, y$ and $z$ principal directions. The hydrodynamic density, $\rho$, and the macroscopic velocity, $\mathbf{u}$, are determined in terms of the particle distribution functions from $\rho=\sum_{i} f_{i}=\sum_{i} f_{i}^{(e q)}$ and $\rho \mathbf{u}=\sum_{i} \mathbf{e}_{i} f_{i}=\sum_{i} \mathbf{e}_{i} f_{i}^{(e q)}$.

\section{Simulations}

In all simulations the flow rate in the tube is computed from a measured aortic pressure at the entrance. Its Fourier terms, up to the 8th harmonic, are used to set a suitable pressure gradient for obtaining an average Reynolds number $R_{e}=\frac{U D}{\nu}=590$ and a Womersley parameter $\alpha=R \sqrt{\frac{\omega}{\nu}}=16$, where $R=$ $D / 2$ is the radius of the tube, $\omega=2 \pi / T$ is the angular frequency and $T=$ $1 / f$ is the period, with $f$ being the number of heart beats per second. For details see [3]. Pressure boundary conditions are used for the inlet and the outlet boundaries and, for the walls, either the bounce-back on the links (BBL) or the Bouzidi boundary condition (BBC) [5] is used. We have performed three different categories of simulations of systolic flow in a 3D rigid tube benchmark and one simulation for the aortic bifurcation. The first set of simulations compares BBL with BBC. The second set deals with error behavior and the third set investigates the convergence behavior. 7

\subsection{Influence of the Wall Boundary Conditions}

A wall boundary condition is needed to evaluate distributions coming from solid boundaries, while inlet/outlet conditions are needed to drive the flow. In this work, we investigate the error behavior for a complete systolic cycle which contains at least 16 harmonic terms. Moreover, we compare the error behavior for 
the two boundary conditions at a fixed Mach number in order to know how much gain we get from using a more accurate, rather sophisticated boundary condition, such as the BBC, over the less accurate but simple BBL.

The diameter of the tube is represented by 74 lattice nodes and the tube length is $L=148$ lattice nodes. First, BBL is used to simulate systolic flow in the tube. The simulation parameters are set to yield the required Womersley and Reynolds numbers which are kept fixed to the values mentioned above. For this simulation, $T=2000$, pressure gradient $G=1.1 \times 10^{-5}, \tau=0.55$ and $A$ is amplitude of Furie series. Samples of obtained velocity profiles at different times of the systolic cycle are shown in Fig. 1(a) compared to the real part of the analytical Womersley solutions [8]. The average Mach number is 0.05 for this simulation. The relative error in velocity at each time step is defined by

$$
E v=\frac{\sum_{i=0}^{n}\left|\mathbf{u}_{t h}\left(\mathbf{x}_{i}\right)-\mathbf{u}_{l b}\left(\mathbf{x}_{i}\right)\right|}{\sum_{i=1}^{n}\left|\mathbf{u}_{t h}\left(\mathbf{x}_{i}\right)\right|}
$$

where $\mathbf{u}_{t h}\left(\mathbf{x}_{i}\right)$ is the analytical solution for the axial velocity and $\mathbf{u}_{l b}\left(\mathbf{x}_{i}\right)$ is the velocity obtained from the LBGK simulations. The bounce back on the links yields a time-averaged error of 0.11 at a Mach number of 0.05 for this specific simulation. The set of simulations is conducted for the same simulation parameters but using the BBC boundary condition. The agreement with analytical solutions enhances significantly, as shown in Fig. 1(b) and the average error reduces to approximately 0.03 .

\section{Grid Refinement}

For pulsatile flow in rigid tubes, it is more convenient to tune the lattice viscosity $\nu$, the Mach number $M$, the diameter $D$ of the tube and the period $T$ of the

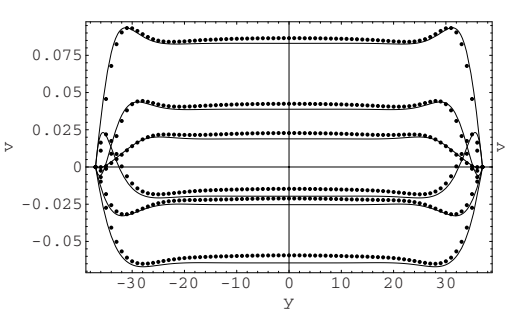

(a)

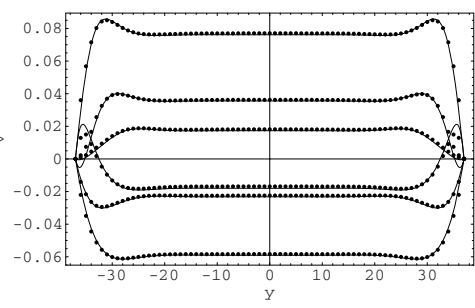

(b)

Fig. 1. Obtained samples of velocity profiles (dots) in lattice units during the systolic cycle in a 3D tube, compared to the analytical Womersley solution (lines) with: (a) $\mathrm{BBL}$ and (b)BBC wall boundary conditions 
pulse. Changes in any of these parameters will result in changes in the space and time resolutions of the grid, and accordingly, the Mach and the Knudsen numbers. In LBGK simulations the error behavior is influenced by the Mach number, $M=\frac{U}{c_{s}}$, and the Knudsen number $\epsilon \sim(2 \tau-1) / D$ which approximates the mean free path in the lattice BGK method. The compressibility error of lattice BGK is given by [9]

$$
\phi=\delta_{x}^{2} R_{e}^{2} \nu^{2} c^{2} \partial_{t} \rho
$$

increases with increasing Reynolds and Mach numbers, since $R_{e}=M D c_{s} / \nu$ and goes to zero as $\tau=1$ where the method behaves the same as finite difference methods. As a function of Womersley number, the compressibility error can be written as

$$
\phi=\frac{2 \delta_{x}^{2} c_{s} c^{2}}{\pi} \frac{\alpha^{2} M D}{S t} \partial_{t} \rho
$$

where $S t=D f / U$ is the Strouhal number. In our simulations, the dimensionless hydrodynamic numbers $(R e, \alpha$ and $S t)$ and the Mach or Knudsen numbers are all fixed. This implies that the grid must be refined. There are three different ways to do this;

1. fixed $M$ method: in which the the diameter $D$, the period $T$ and the viscosity $\nu$ are changed while keeping fixed the Mach number.

2. fixed $\tau$ method: by changing the diameter $D$, the period $T$ and the Mach number $M$ while keeping fixed the lattice viscosity $\nu$.

3. fixed $D$ method : by keeping fixed the diameter $D$ while changing the viscosity, period and the Mach number.

Table 1. Relative changes in simulation parameters under fixed Reynolds and Womersley numbers with respect to an $n$ times change in one of the parameters of a reference simulation

\begin{tabular}{lcccccccc}
\hline Lattice Parameter & $\mathrm{D}^{\prime} / \mathrm{D}$ & $\nu^{\prime} / \nu$ & $T^{\prime} / T$ & $U^{\prime} / U$ & $\delta x^{\prime} / \delta x$ & $\delta t^{\prime} / \delta t$ & $M^{\prime} / M$ & $\epsilon^{\prime} / \epsilon$ \\
\hline Fixed $D$ & 1 & $1 / n$ & $n$ & $1 / n$ & 1 & $1 / n$ & $1 / n$ & $1 / n$ \\
Fixed $\tau$ & $n$ & 1 & $n^{2}$ & $1 / n$ & $1 / n$ & $1 / n^{2}$ & $1 / n$ & $1 / n$ \\
Fixed $M$ & $n$ & $n$ & $n$ & 1 & $1 / n$ & $1 / n$ & 1 & 1 \\
\hline
\end{tabular}

The effects of these changes on the grid resolution are shown in Table 1, in which we assume an $n$ times change in one of the parameters and compute the corresponding changes in the other parameters to return the fixed $R_{e}$ and $\alpha$. The fixed $M$ method does not involve reduction of the Mach number, which is a major contributer to the error when considering time dependent flows and, therefore, it is not attractive in this study. 
Table 2. Simulation parameters with respect to the reference simulation for which $\tau=1, M=0.50$ and $\epsilon=1 / 74$. The average error Eav associated with each set is shown for BBL, BBC1 and BBC2 boundary conditions

\begin{tabular}{lccc}
\hline $\mathrm{T}$ & 200 & 2000 & 20000 \\
\hline $\mathrm{n}$ & 1 & 10 & 100 \\
$\tau$ & 1 & 0.55 & 0.505 \\
$\frac{G^{\prime}}{G}$ & 1 & $1 / 100$ & $1 / 10000$ \\
$\frac{M^{\prime}}{M}$ & 1 & $1 / 10$ & $1 / 100$ \\
$\frac{\epsilon^{\prime}}{\epsilon}$ & 1 & $1 / 10$ & $1 / 100$ \\
$\frac{\epsilon^{\prime} M^{\prime}}{\epsilon M}$ & 1 & $1 / 100$ & $1 / 10000$ \\
Eav, BBL & instable & 0.120 & 0.027 \\
Eav, BBC1 & 0.0627 & 0.0352 & 0.0253 \\
Eav, BBC2 & 0.0615 & 0.0102 & instable \\
\hline
\end{tabular}

Accuracy and Performance with the Fixed $D$ Method. In all simulations, the system is initialized from rest and the simulation ends after 40-50 complete periods. The BBL, BBC1 (first order Bouzidi) and BBC2 (second order Bouzidi) are used separately for each parameter set to end up with 9 simulations. The simulations are performed on 8 nodes of a Beowulf cluster using slice decomposition.

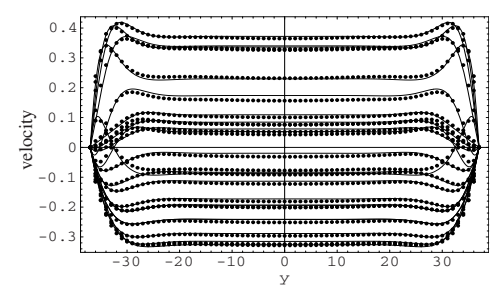

Fig. 2. Velocity profiles at $M=0.50$ using the BBC1 boundary condition with overall average error of about 0.07 , still less than the BBL results at a 10 times lower Mach number. The system is instable with the BBL at this Mach number

The mean time per iteration is 0.2 seconds. Samples of BBC1 obtained velocities and shear stress profiles during the systolic cycle are shown in Fig. 2 for $M=0.5$, compared to the analytical Womersley solutions. Although $M$ is relatively high, the agreement with the analytical solution is still better than those obtained by a 10 times smaller Mach number with BBL shown in Fig. 1(a). The last three rows in Table 2 list the average error associated with the three wall boundary conditions. 
Accuracy and Performance with the Fixed $\tau$ Method. In order to reduce simulation time, it is necessary to have a large time-step in a coarse grid at a high Mach number. We use the fixed $\tau$ method to perform a set of simulations in which the period is set to the smallest possible value that leads to a stable solution on the coarsest grid. Then the corresponding values for the pressure gradient and

Table 3. Simulation parameters used to enhance the spatial resolution. The mean relative error, Eav, is listed for each case

\begin{tabular}{lcccc}
\hline$D$ & 21 & 65 & 105 & $21 n$ \\
\hline$n$ & 1 & 3 & 5 & $\mathrm{n}$ \\
$T$ & 40 & 360 & 1000 & $40 n^{2}$ \\
$\frac{G^{\prime}}{G}$ & 1 & $1 / 27$ & $1 / 125$ & $1 / n^{3}$ \\
$\frac{M^{\prime}}{M}$ & 1 & $1 / 3$ & $1 / 5$ & $1 / n$ \\
$\delta t$ & 1 & $1 / 9$ & $1 / 25$ & $1 / n^{2}$ \\
Eav, BBL & 0.2412 & 0.1189 & 0.0262 & \\
Eav, BBC & 0.2301 & 0.0557 & 0.0262 & \\
Eav, BBC2 & instable & 0.0560 & 0.0266 & \\
\hline
\end{tabular}

the relaxation parameter are set to yield the desired Womersley and Reynolds numbers. The convergence behavior is studied by grid refinement in both $\delta_{x}$ and $\delta_{t}$, as explained in Table 1 . The simulation parameters are listed in Table 3 together with obtained average errors associated with the three used boundary conditions. As it is shown in Fig. 3, at least second order convergence behavior is guaranteed with this method. Moreover, solutions with periods smaller than the fixed $D$ method are stable and therefore the simulation time is less, but it scales as $n^{2}$. The convergence behavior as a function of time for this method is shown in Fig. 4 which shows the difference between the analytical and obtained velocity profiles at different simulation times. In this figure, the method converges to a reasonable accuracy after 40 complete periods, similar to the fixed $D$ method, but with a major computational gain, since the length of the period is smaller (i.e. $\delta_{t}$ is larger). This figure also illustrates that the error is localized near to the walls, where large gradients exist, and it does not enhance noticeably near to the walls on the same grid. Table 4 lists the error dependence as a function of simulation times for $\mathrm{BBL}, \mathrm{BBC} 1$ and $\mathrm{BBC} 2$ boundary conditions for a tube with $D=65$ lattice nodes. In conclusion, this method is computationally more feasible than the fixed $D$ method.

\subsection{Convergence Behavior}

Convergence to steady state of the lattice Boltzmann method is significantly affected by two local processes; initialization and boundary conditions. In this section, we focus on the influence of initial and boundary conditions on the convergence behavior. 
Table 4. Mean, variance and mean deviation of the Relative error for BBL, BBC boundary conditions with $D=65$ lattice nodes

\begin{tabular}{lccc}
\hline Boundary condition & Mean error (Eav) & Variance & Mean deviation \\
\hline BBL & 0.1189 & 0.0013 & 0.0219 \\
BBC & 0.0557 & 0.0027 & 0.0352 \\
BBC2 & 0.0560 & 0.0027 & 0.0350 \\
\hline
\end{tabular}

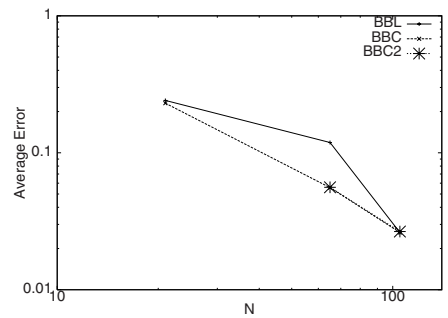

Fig. 3. Convergence behavior obtained by reducing the grid spacing $n$ times, time-step $n^{2}$ times and increasing the period $n^{2}$ times, for the BBL, BBC and BBC2 boundary conditions as a function of grid points. The relaxation parameter is kept constant and the body force is reduced $n^{3}$ times to return the same Reynolds and Womersley parameters at $R e=$ and $\alpha=16$

Convergence and Walls Boundary Conditions. For the walls, bouncebacks and curved boundaries can be recognized. The bounce back is a very efficient boundary condition since it only involves a single memory swapping process for each relevant distribution on each node on the surface of the simulated object. For all curved boundaries, the exact position of the walls is determined at least once if the boundary is fixed and needs to be computed dynamically for moving boundaries. This is more costly than using the bounce back rule. [7]

Inlet and Outlet Conditions. For non-periodic geometry, inlets and outlets need to be treated differently in the following manner:

- Velocity and pressure : assign one and compute the other [10, assign both (only for inlets) extrapolate or no flux normal to the walls (only for outlets).

- Unknown distributions: compute explicitly [10], set to their equilibrium, copy from nearest neighbors, interpolate or extrapolate.

For the first item at least 15 additions and two multiplications are needed per node on the boundary and therefore is at least 15 times more expensive than periodic boundary conditions. A reasonable choice is then to assign pressure and compute velocity at the inlet, no-flux at the outlets and set the unknown distributions to their equilibrium values. If the outlets are far enough from inflow, copying from upstream would be the most efficient outlet condition. 


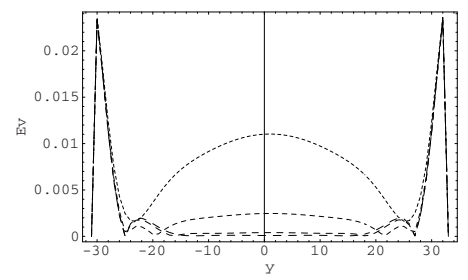

Fig. 4. Local deviations from Analytical solutions, $\delta E$, computed for the velocity field at $t=20 T$ (top Curve), 30T, $40 T$ and $50 T$ (bottom curve). The diameter of the tube is represented by 65 nodes and the period is $T=360$ sampling points

\section{Conclusions}

We have shown that the lattice Boltzmann BGK method is an accurate and efficient method as a solver for time-dependent flows. Different methods for performing time dependent flows at fixed simulation parameters are tested in terms of accuracy and performance. An aortic pressure is used as an inlet condition to drive the flow in a $3 \mathrm{~d}$ rigid tube and the Womersley solution is recovered to an acceptable accuracy. The influence of walls, inlet and outlet boundary conditions on accuracy and performance is studied in details as a function of Mach and Knudsen numbers.

Acknowledgments. We acknowledge Prof. Peter M.A. Sloot for his support and helpfull discussions. This work was partially funded by the Steunfonds Soedanese Studenten, Leiden, The Netherlands, and by the Dutch National Science Foundation, NWO, through the DIME-Token2000 project.

\section{References}

1. Xiaoyi He and Li-Shi Luo, J. Stat. Phys. 88, 927 (1997); Q. Zou, S. Hou, S.Chen and G. D. Doolen, J. Stat. Phys. 81, 35 (1995); Guo, Shi and Wang, J. Comp. Phys. 165, 288 (2000).

2. M. Krafczyk, M. Cerrolaza, M. Schulz, E. Rank, J. Biomechanics 31, 453 (1998).

3. A. M. Artoli, A. G. Hoekstra, and P. M. A. Sloot, Int. J. Mod. Phys. C, 13 No. 8, 1119 (2002).

4. O. Fillippova and D. Hänel, J. Comp. Phys. 147, 219 (1998).

5. M. Bouzidi, M. Fidouss and P. Lallemand, Phys. Fluids 13, 3452 (2001).

6. P. L. Bhatnagar, E. P. Gross, and M. Krook, Phys. Rev. A, 94, 511 (1954).

7. A. M. Artoli, Mesoscopic Computational Haemodinamics, PhD thesis, University van Amsterdam, The Netherlands (2003).

8. C. Pozrikidis, Introduction to Theoretical and Computational Fluid Dynamics, OUP, (1997).

9. D. Holdych, D. Noble, J. G. Georgiadis, and R. O. Buckius, Proceedings of the 11th international conference of Discrete simulation of fluid dynamics and soft condensed matter, Shanghai, China, August 5-9 (2002).

10. Q. Zao and X. He, Phys. fluids 9, 1591(1997). 